\title{
Análise de cor e aceitabilidade de molho agridoce de abacaxi com pimenta elaborado com diferentes espessantes
}

Color and acceptability analysis of pineapple sauce in pineapple with pepper made with diferente thickeners

Análisis de color y aceptabilidad de salsa de piña em piña com pimenta preparada com diferentes espesantes

Luíz Guilherme Malaquias da Silva ORCID: https://orcid.org/0000-0003-4958-5161 Instituto Federal de Educação, Ciência e Tecnologia do Sul de Minas Gerais, Brasil E-mail: luizgms98@gmail.com

Henrique Costa Silva Zandomenegui ORCID: https://orcid.org/0000-0002-7081-6131 Instituto Federal de Educação, Ciência e Tecnologia do Sul de Minas Gerais, Brasil E-mail: hzandomenegui@gmail.com

Brenda Regina Aires Vieira

ORCID: https://orcid.org/0000-0001-8194-4940 Instituto Federal de Educação, Ciência e Tecnologia do Sul de Minas Gerais, Brasil E-mail: bso95.heck@hotmail.com

Marcela Costa Rocha

ORCID: https://orcid.org/0000-0002-2382-8447 Instituto Federal de Educação, Ciência e Tecnologia do Sul de Minas Gerais, Brasil E-mail: marcela.rocha@ifsuldeminas.edu.br

Aline Manke Nachtigall

ORCID: https://orcid.org/0000-0002-9691-0361 Instituto Federal de Educação, Ciência e Tecnologia do Sul de Minas Gerais, Brasil

E-mail: aline.manke@ifsuldeminas.edu.br

\begin{abstract}
Resumo
A demanda elevada por alimentos saudáveis é um fator relevante para o desenvolvimento de produtos alimentícios. Neste sentido, os vegetais são ricos em nutrientes que lhes conferem propriedades funcionais. Portanto, objetivou-se elaborar um molho para carnes, de abacaxi com pimenta, e avaliar a sua coloração e aceitabilidade sensorial. Foram elaboradas três formulações com as mesmas condições de processamento e variação nos espessantes empregados (F1 Goma Xantana; F2 - Goma Carragena; F3 - Carboximetilcelulose). Os molhos foram elaborados pela mistura dos ingredientes a frio. Avaliaram-se os parâmetros instrumentais de cor $\left(\mathrm{L}^{*}, \mathrm{a}^{*}, \mathrm{~b}^{*}, \mathrm{H}^{\circ}, \mathrm{C}^{*}\right)$, com colorímetro Minolta CR400. O teste de aceitação dos atributos aroma, cor, sabor, consistência e aspecto global, foi realizado fazendo uso de uma escala hedônica de 9 pontos e o teste de intenção de compra com escala de atitude de 5 pontos, ambos com 80 consumidores. Os molhos apresentaram coloração amarela, com maior luminosidade $(45,38)$ e intensidade $(39,55)$ de cor observada no molho elaborado com Goma Carragena, enquanto a maior tonalidade $(85,02)$ foi percebida no molho produzido com Goma Xantana. Os molhos apresentaram boa aceitação para todos os atributos, com índice de aceitabilidade superior a 70\%, exceto para a consistência do molho elaborado com Goma Carragena (67\%). Não foram verificadas diferenças significativas entre a intenção de compra das amostras (F1- 3,70; F2- 3,54; F3- 3,43). Portanto, o estudo demonstra a importância da escolha do espessante para a elaboração de molhos e a necessidade de adequação da formulação elaborada com a Goma Carragena.
\end{abstract}

Palavras-chave: Consistência; Colorimetria; Analise sensorial; Desenvolvimento de produtos; Alimentos funcionais.

\section{Abstract}

The high demand for healthy food is a relevant factor for the development of food products. In this sense, vegetables are rich in nutrients that give them functional properties. Therefore, the objective was to prepare a meat sauce, made of pineapple with pepper, and to evaluate its color and sensory acceptability. Three formulations were prepared with the same processing conditions and variation in the thickeners used (F1 - Xanthan Gum; F2 - Carragena Gum; F3 Carboxymethylcellulose). The sauces were made by mixing the ingredients cold. Instrumental color parameters $(\mathrm{L} *$, a $*, \mathrm{~b} *, \mathrm{H}^{\circ}, \mathrm{C} *$ ) were evaluated with a Minolta CR400 colorimeter. The acceptance test of the attributes of aroma, color, flavor, consistency and global aspect was performed using a 9-point hedonic scale and the purchase intention test with 
a 5-point attitude scale, both with 80 consumers. The sauces showed yellow color, with greater luminosity (45.38) and intensity (39.55) of color observed in the sauce elaborated with Goma Carragena, while the greater hue (85.02) was perceived in the sauce produced with Goma Xantana. Sauces showed good acceptance for all attributes, with an acceptability index greater than $70 \%$, except for the consistency of the sauce made with Goma Carragena (67\%). There were no significant differences between the intention to purchase the samples (F1- 3.70; F2- 3.54; F3- 3.43). Therefore, the study demonstrates the importance of choosing a thickener for the preparation of sauces and the need to adapt the formulation prepared with Carragena Gum.

Keywords: Consistency; Colorimetry; Sensory analysis; Product Development; Functional foods.

\section{Resumen}

La alta demanda de alimentos saludables es un factor relevante para el desarrollo de productos alimenticios. En este sentido, las verduras son ricas en nutrientes que les confieren propiedades funcionales. Por tanto, el objetivo fue preparar una salsa de carne, a base de piña con pimienta, y evaluar su color y aceptabilidad sensorial. Se prepararon tres formulaciones con las mismas condiciones de procesamiento y variación en los espesantes utilizados (F1 - Goma de xantano; F2 - Goma de Carragena; F3 - Carboximetilcelulosa). Las salsas se elaboraron mezclando los ingredientes en frío. Los parámetros de color instrumentales ( $\mathrm{L}^{*}, \mathrm{a} *, \mathrm{~b}^{*}, \mathrm{H}^{\circ}, \mathrm{C} *$ ) se evaluaron con un colorímetro Minolta CR400. La prueba de aceptación de los atributos de aroma, color, sabor, consistencia y aspecto global se realizó mediante una escala hedónica de 9 puntos y la prueba de intención de compra con una escala de actitud de 5 puntos, ambas con 80 consumidores. Las salsas mostraron color amarillo, con mayor luminosidad $(45,38)$ e intensidad $(39,55)$ de color observada en la salsa elaborada con Goma Carragena, mientras que la mayor tonalidad $(85,02)$ se percibió en la salsa elaborada con Goma Xantana. Las salsas mostraron buena aceptación para todos los atributos, con un índice de aceptabilidad superior al 70\%, a excepción de la consistencia de la salsa elaborada con Goma Carragena (67\%). No hubo diferencias significativas entre la intención de compra de las muestras (F1-3,70; F2- 3,54; F3- 3,43). Por ello, el estudio demuestra la importancia de elegir un espesante para la preparación de salsas y la necesidad de adecuar la formulación elaborada con Goma de Carragena.

Palabras clave: Consistencia; Colorimetría; Análisis sensorial; Desarrollo de produtos; Alimentos funcionales.

\section{Introdução}

Ao longo dos anos vem se incorporando o processo de inovação em produtos alimentícios, assim sendo, o desenvolvimento e aperfeiçoamento de novos produtos têm adquirido relevância, devido ao fato da crescente demanda exigida pelos consumidores por alimentos que apresentem, além da alta qualidade sensorial e nutricional, benefícios associados à saúde (Santos, 2019).

Os molhos além de acompanharem pratos de todos os tipos são responsáveis por conferir textura às receitas, sendo suas principais funções introduzir sabores complementares ou de contraste, acrescentar umidade ou suculência e auxiliar no interesse visual do prato (IAC, 2011).

O abacaxi (Ananas comosus) é uma fruta de clima tropical, sendo o Brasil o segundo maior produtor, responsável por 12\% de toda a produção mundial (FAOSTAT, 2018), em 2018, produziu-se 1.766.986 toneladas, com área de cultivo de 71.553 ha (IBGE, 2019). A fruta possui propriedades nutricionais que auxiliam no bom funcionamento imunológico, melhora a função intestinal e facilita a digestão pela ação da bromelina, além de regular a atividade muscular do coração e ser um excelente diurético (Neres, Souza \& Bezerra, 2015; Borges et al., 2020).

As pimentas do gênero Capsicum, pertencentes à família Solanaceae, são empregadas em alimentos devido as suas grandes variedades de cores e sabores, auxiliando na conservação dos alimentos ao evitar a contaminação por fungos e bactérias. Segundo Lainett (2017) possuem antioxidantes naturais como vitaminas A e E, além de flavonoides, que são substâncias que atuam contra a ação dos radicais livres e previnem doenças crônicas como as cardíacas, o diabetes, o câncer, além de evitar o envelhecimento precoce. De acordo com Véras (2010) a pimenta atua como vasodilatadora, estimulando a produção de endorfina no cérebro e é fonte de vitamina $\mathrm{C}$. O efeito termogênico eleva a temperatura do organismo, aumentando o gasto calórico, e consequentemente pode auxiliar no emagrecimento (Carvalho et al., 2009).

Os espessantes são aditivos que melhoram a textura e a consistência dos alimentos processados, sem modificar de forma significativa suas propriedades (Aun et al., 2011). O uso de diferentes espessantes pode conferir viscosidade com diferentes 
graus de aceitação pelos consumidores, devido à textura percebida ao degustarem esses produtos. Portanto, neste estudo testouse o efeito de três espessantes na aceitação de molho agridoce de abacaxi com pimenta.

A Goma Xantana é um polissacarídeo de extrema importância comercial, sua elevada utilização em alimentos é devido a sua elevada viscosidade em baixas concentrações e a sua estabilidade (FIB, 2010). A Goma Carragena possui capacidade de ligar-se a água e formar sistemas coloidais, com perfil pseudoplástico, sendo suas características reológicas úteis para modificar características sensoriais e também nutricionais dos alimentos (Blackmore \& Harpell, 2010; Valdez, 2012). Já a Carboximetilcelulose é um polímero aniônico, altamente higroscópico, solúvel em água e insolúvel em solventes orgânicos que possui boa resistência ao ataque microbiano. De acordo com Funami et al. (2008) apresenta-se útil no controle da sinérese por reter água.

Diante do exposto, o estudo teve por objetivo a elaboração de um molho agridoce de abacaxi com pimenta, empregando diferentes espessante, e avaliar a influência destes na cor a na aceitação dos mesmos.

\section{Metodologia}

Para a realização deste estudo, utilizou-se uma metodologia de natureza quantitativa em modelo experimental. Pereira et al. (2018) caracterizam o método quantitativo, como aquele em que há coleta de dados quantitativos ou numéricos por meio do uso de medições de grandezas, as quais são analisados através de cálculos matemáticos.

\subsection{Elaboração dos molhos}

Os espessantes empregados na elaboração dos molhos foram cedidos por uma empresa de molhos e especiarias de Machado/MG e os demais ingredientes foram adquiridos no comércio local. Os molhos foram elaborados na Cozinha Experimental do Instituto Federal Educação, Ciência e Tecnologia do Sul de Minas Gerais IFSULDEMINAS - Campus Machado, sendo desenvolvidos três molhos agridoces de abacaxi com pimenta, com diferentes espessantes (F1 - Goma Xantana; F2 - Goma Carragena; F3 - Carboximetilcelulose).

Os molhos foram elaborados seguindo as Boas Práticas de Fabricação (PBF), com três repetições, empregando a seguinte formulação base: polpa de abacaxi (500 g), açúcar (200 g), água (200 g), vinagre triplo de álcool (20 g), amido prégelatinizado (5 g), espessante ( $4 \mathrm{~g})$, molho de pimenta (3,5 g), cúrcuma ( $\mathrm{g}$ g), ácido cítrico (3 g) e sorbato de potássio (1,4 g).

O preparo dos molhos foi a frio com as matérias primas previamente refrigeradas. Inicialmente, a polpa de abacaxi foi homogeneizada e padronizada em $11,90^{\circ}$ Brix. Na sequência os ingredientes foram pesados e separados em três mix a saber: Mix 1 composto por água e açúcar, Mix 2 formado por vinagre, polpa de abacaxi e ácido cítrico e o Mix 3 por água, sorbato de potássio, açúcar, espessantes, amido pré-gelatinizado e molho de pimenta. Utilizou-se um mixer da Mondial - Power Mixer (500W de potência) para a homogeneização dos ingredientes. Primeiramente, os Mix foram homogeneizados separadamente por 3 min, sendo na sequência misturados o Mix 1 com o Mix 2 por 5 min., em seguida acrescentou-se à mistura o Mix 3 que foi homogeneizado por mais $5 \mathrm{~min}$. Os molhos foram armazenados em recipientes plásticos de $500 \mathrm{ml}$, lacrados com lacre metálicos e armazenados em refrigeração até o momento da análise.

\subsection{Análise de cor}

Para determinar a cor dos molhos, realizou-se no Laboratório de Bromatologia do IFSULDEMINAS - campus Machado, as leituras dos valores $\mathrm{L}^{*}, \mathrm{a}^{*}, \mathrm{~b}^{*}, \mathrm{H}^{\circ} \mathrm{e} \mathrm{C}^{*}$ diretamente na superfície das três formulações de molho, utilizando colorímetro $\mathrm{CR}$ 400 (Konica Minolta®), com iluminante D65, ângulo de observação de $2^{\circ}$ e no sistema de cor CIEL*a*b* (Minolta, 1998). 


\subsection{Teste com consumidores}

As análises foram realizadas no Laboratório de Análise Sensorial do IFSULDEMINAS - campus Machado. A aceitabilidade dos molhos foi analisada, um dia após o processamento, por 80 consumidores, de ambos os sexos, com idade entre 18 e 60 anos, em cabines individuais, com luz branca, onde as amostras de molhos (cerca de $2 \mathrm{~g}$ ) foram apresentadas de forma aleatória e balanceada, em bandeja de isopor de fundo branco, servidas em recipiente plástico codificados com três dígitos aleatórios, acompanhados de pedaços de frango grelhados que foram utilizados como veículo para a prova dos molhos e copo de água para limpeza das papilas gustativas.

Os consumidores receberam uma ficha para analisarem a aceitação dos atributos aroma, cor, consistência, sabor e aspecto global fazendo uso de uma escala hedônica estruturada de 9 pontos ( 9 - gostei extremamente a 1- desgostei extremamente) e, para avaliar a intenção de compra uma escala de atitude estruturada mista de 5 pontos ( 5 - certamente compraria e 1 - certamente não compraria), de acordo com Reis e Minin (2006). O estudo foi submetido e aprovado pelo Comitê de ética do Instituto Federal de Educação, Ciência e Tecnologia de Minas Gerais e está cadastrado sob o CAAE 68943717.8.0000.5143.

O cálculo do índice de aceitabilidade (IA) foi realizado, de acordo com metodologia de Monteiro (1984), que considera a razão entre a nota média e a nota máxima dada ao produto multiplicada por 100.

\subsection{Análise estatística}

O experimento foi conduzido em delineamento em blocos casualizado (DBC). Foi realizada a análise de variância e comparação de médias pelo teste de Tukey, quando necessária, com auxílio do Software SISVAR, ao nível de 5\% de significância (Ferreira, 2008).

\section{Resultados e Discussão}

Ao analisar a Tabela 1, observa-se que os espessantes influenciaram significativamente a cor dos molhos, em relação a todos os parâmetros instrumentais analisadas.

Tabela 1. Valores médios e desvio padrão para $\mathrm{L}^{*}, \mathrm{a}^{*}, \mathrm{~b}^{*}, \mathrm{H}^{\circ}$ e $\mathrm{C}^{*}$, para as formulações dos molhos.

\begin{tabular}{cccc}
\hline Parâmetros & $\begin{array}{c}\text { Molho com } \\
\text { Xantana }\end{array}$ & Molho com Carragena & $\begin{array}{c}\text { Molho com } \\
\text { Carboximetilcelulose }\end{array}$ \\
\hline $\mathrm{L}^{*}$ & $45,38 \mathrm{a} \pm 0,02$ & $38,47 \mathrm{~b} \pm 0,06$ & $37,18 \mathrm{c} \pm 0,06$ \\
$\mathrm{a}^{*}$ & $4,11 \mathrm{a} \pm 0,55$ & $2,96 \mathrm{c} \pm 0,37$ & $2,99 \mathrm{~b} \pm 0,43$ \\
$\mathrm{~b}^{*}$ & $39,34 \mathrm{a} \pm 0,24$ & $34,01 \mathrm{~b} \pm 0,01$ & $27,89 \mathrm{c} \pm 0,28$ \\
$\mathrm{H}^{\circ}$ & $84,03 \mathrm{~b} \pm 0,02$ & $85,02 \mathrm{a} \pm 1,20$ & $83,88 \pm \mathrm{c} 0,58$ \\
$\mathrm{C}^{*}$ & $39,55 \mathrm{a} \pm 1,04$ & $34.13 \mathrm{~b} \pm 0,51$ & $28,04 \mathrm{c} \pm 0,61$ \\
\hline
\end{tabular}

*Médias seguidas da mesma letra na linha não diferem entre si, pelo Teste de Tukey (p< 0,05). Fonte: Própria (2021).

Os valores de $\mathrm{L}^{*}$ indicam a luminosidade, uma vez que os valores variam do preto ao branco (Souza, 2018). A formulação com Goma Xantana apresentou a maior luminosidade ( $\left.\mathrm{L}^{*}\right)$, 45,38 seguida pela formulação com Goma Carragena $(38,47)$ e CMC $(37,18)$. A quantidade empregada de cada espessante pode ter influenciado esse aspecto devido à concentração de densidade proporcionado pelos tratamentos.

Além da luminosidade a formulação de molho com Goma Xantana (F1) apresentou maior valor para a* $(4,11)$, b* $(39,34)$ e $C^{*}(39,55)$ ou seja, com maior intensidade em comparação com as demais formulações, enquanto a formulação de molho com Goma Carragena (F2) apresentou maior valor para $\mathrm{H}^{\circ}(82,02)$, sendo a formulação com maior tonalidade, visto que no geral os molhos apresentaram cores claras e vivas, o que colaborou para a aceitação da cor entre os provadores. Essa indicação 
de tonalidade é devido as características das matérias primas, com coloração clara (abacaxi) e viva (pimenta), além da adição de componentes pungentes como a cúrcuma que possui características similares de coloração viva. O parâmetro $\mathrm{C}^{*}$ refere-se à intensidade enquanto $\mathrm{o} \mathrm{H}^{\circ}$ refere-se à tonalidade da coloração, que aumenta a partir de zero, conforme $\mathrm{a}^{*}$ e $\mathrm{b}^{*}$ aumentam (Minolta, 1998).

Os dados da aceitabilidade dos molhos formulados encontram-se na Tabela 2, na qual pode-se observar que as amostras foram classificadas entre os termos hedônicos "gostei ligeiramente" e "gostei moderadamente".

Tabela 2 Valores médios e desvio padrão para a aceitação dos atributos aroma, cor, consistência, sabor e aspecto global dos molhos

\begin{tabular}{lccc}
\hline Atributos & $\begin{array}{c}\text { Molho com } \\
\text { Xantana }\end{array}$ & $\begin{array}{c}\text { Molho com } \\
\text { Carragena }\end{array}$ & $\begin{array}{c}\text { Molho com } \\
\text { Carboximetilcelulose }\end{array}$ \\
\hline Aroma & $7,14 \mathrm{a} \pm 1,57$ & $6,65 \mathrm{a} \pm 1,79$ & $6,67 \mathrm{a} \pm 1,76$ \\
Cor & $7,34 \mathrm{a} \pm 1,39$ & $7,08 \mathrm{a} \pm 1,42$ & $7,29 \mathrm{a} \pm 1,29$ \\
Consistência & $7,01 \mathrm{a} \pm 1,81$ & $6,07 \mathrm{~b} \pm 1,76$ & $6,70 \mathrm{ab} \pm 1,62$ \\
Sabor & $7,14 \mathrm{a} \pm 1,57$ & $6,75 \mathrm{a} \pm 1,75$ & $6,67 \mathrm{a} \pm 1,62$ \\
Aspecto Global & $7,13 \mathrm{a} \pm 1,55$ & $6,70 \mathrm{a} \pm 1,47$ & $7,01 \mathrm{a} \pm 1,47$ \\
\hline
\end{tabular}

*Médias seguidas da mesma letra na linha não diferem entre si, pelo Teste de Tukey (p<0,05). Fonte: Própria (2021).

Os distintos espessantes não interferiram na aceitação dos atributos aroma, cor, sabor e aspecto global dos molhos agridoces de abacaxi com pimenta (Tabela 2). Já com relação ao atributo consistência, percebem-se diferenças entre as médias de aceitação, onde o molho elaborado com a Goma Xantana obteve média de 7,01, não diferindo do molho elaborado com a Carboximetilcelulose (6,70), que por sua vez foi semelhante ao molho elaborado com a Goma Carragena $(6,07)$.

A diferença de consistência entre as amostras desse estudo pode ser atribuída às características intrínsecas dos espessantes empregados, que foram utilizados na mesma proporção em relação à polpa de abacaxi para todas as formulações. Portanto, percebe-se a necessidade de ajustar as concentrações dos espessantes, levando-se em consideração o seu comportamento no meio empregado.

Martins e Alves (2017) ao desenvolver molho chutney de hortaliças e frutas verificaram que é viável a produção deste tipo de produto, indo de encontro ao que o consumidor busca em termos de sabor e atratividade. As autoras observaram boa aceitação, com notas variando de 7,38 a 7,92 na escala hedônica para os chutney elaborados com hortaliças e, notas variando de 7,34 a 7,78 para os chutney elaborados com frutas. No presente trabalho os valores médios de aceitação foram um pouco inferiores, no entanto, a aceitação da cor dos chutney de fruta foi igual à do molho agridoce de abacaxi com pimenta elaborado com a Goma Xantana.

Os valores para a aceitação da consistência são similares ao do estudo de Lainetti (2017), cuja consistência de geleias de abacaxi com pimenta variou entre 6,98 e 8,05. Por outro lado, Rabelo e Nascimento (2018) ao desenvolverem geleias de polpa e casca de abacaxi com gengibre verificaram que os atributos sensoriais avaliados foram classificados entre os termos hedônicos gostei moderadamente e gostei muito (valores médios entre 8 e 9). Os dois estudos com geleias empregaram polpas de abacaxi associada a ingredientes pungentes como a pimenta e o gengibre, similar ao empregado no molho agridoce em estudo.

Na Tabela 3, é demonstrado o Índice de Aceitabilidade (IA\%) dos molhos formulados. 
Tabela 3. Índice de Aceitabilidade para os atributos aroma, cor, consistência, sabor e aspecto global dos molhos

\begin{tabular}{lccc}
\hline Atributos & Molho com Xantana & Molho com Carragena & Molho com CMC \\
\hline Aroma & 79,30 & 73,88 & 74,10 \\
Cor & 81,50 & 78,60 & 81,00 \\
Consistência & 77,88 & 67,44 & 74,44 \\
Sabor & 79,30 & 75,00 & 74,14 \\
Aspecto Global & 79,22 & 74,44 & 77,88 \\
\hline
\end{tabular}

*Fonte: Própria (2021).

De acordo com a Tabela 3, os molhos desenvolvidos apresentaram resultados médios para o aspecto global que correspondem a uma boa aceitação, sendo 79,22\% para o molho elaborado com o espessante Goma Xantana (F1), de 74,44\% para o molho com Carragena (F2) e, de 77,88\% para o molho com Carboximetilcelulose (F3). No entanto, ao avaliar o atributo consistência isoladamente, percebe-se que a aceitação do molho elaborado com Carragena não teria uma aceitação $(67,44 \%)$ no mercado, visto que se recomenda um índice de aceitabilidade igual ou superior a 70\% para lançar um produto.

Quirino (2017) ao desenvolver molhos de polpa do mangostão amarelo obteve semelhantes índices de aceitabilidade (IA), sendo que a formulação com menor teor de polpa resultou em um IA médio de 78,46\% e a com maior teor de 71,33\%. Para o atributo consistência também se verificou semelhanças entre os molhos elaborados com o molho agridoce de abacaxi com pimenta associados a Goma Xantana e a carboximetilcelusose quanto ao índice de aceitabilidade, onde a formulação com maior teor de polpa de mangostão amarelo teve IA de $77,78 \%$ e a com menor teor de 74,22\%.

É valido ressaltar que em relação ao sabor, os provadores em geral gostaram do produto, sendo o segundo atributo com maior valor de índice de aceitabilidade, mesmo ao ser avaliado por consumidores que não têm o costume de consumir abacaxi e pimenta, conforme informações dos mesmos. No entanto, alguns consumidores comentaram que a picância do molho poderia ser mais intensa.

Na Figura 1 pode ser observada a distribuição da frequência de notas para a intenção de compra dos molhos de abacaxi com pimenta.

Figura 1. Histograma da intenção de compra dos molhos agridoces de abacaxi com pimenta elaborados com Goma Xantana.

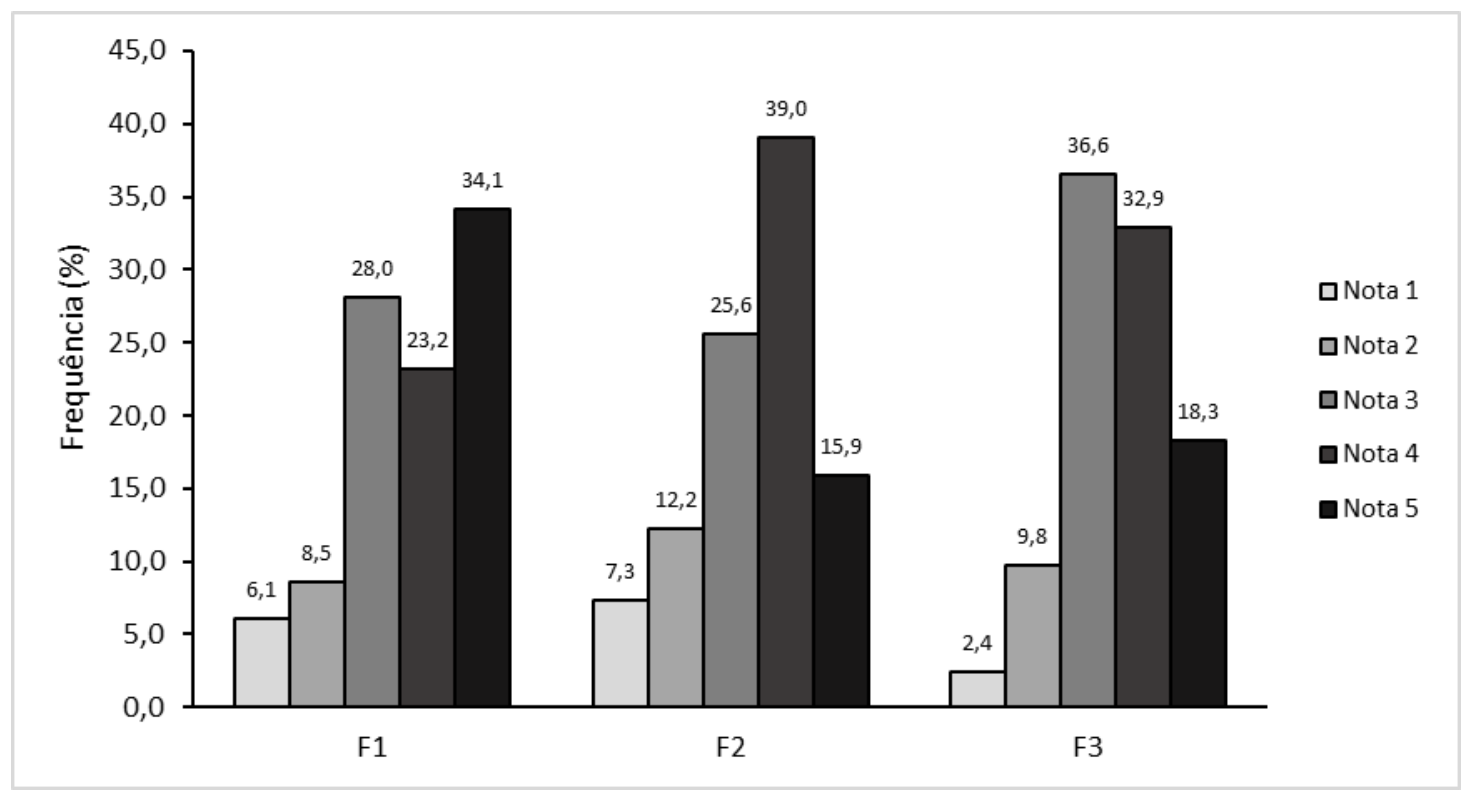

(F1), Goma Carragena (F2) e Carboximetilcelulose (F3). Fonte: Própria (2021). 
Ao avaliar a Figura 1, percebe-se que o molho elaborado com a Goma Xantana (F1) apresentou a maior frequência de notas 5 (com certeza compraria) entre todas as amostras, com 14,6\% de notas abaixo do ponto de indiferença e 57,3\% acima do ponto de indiferença da escala (nota 3). Por sua vez, o molho elaborado com Goma Carragena (F2) apresentou maior frequência de nota 4 (provavelmente compraria), com 19,5\% de notas distribuídas abaixo do ponto de indiferença e 54,9\% acima do ponto de indiferença. Já o molho desenvolvido com Carboximetilcelulose (F3) teve 12,2\% de notas distribuídas abaixo do ponto de indiferença, $38 \%$ no ponto de indiferença e $51,2 \%$ de notas superiores ao ponto de indiferença da escala.

Os valores médios de intenção de compra foram de 3,70 para o molho elaborado com Goma Xantana, 3,54 para o molho produzido com Carboximetilcelulose e de 3,43 para o que empregou a Goma Carragena na formulação. Os molhos foram classificados entre as atitudes de "talvez compraria/talvez não" e "provavelmente compraria".

Alves e Martins (2017) ao estudarem molhos chutney salgados com hortaliças (cenoura e pimentão) e agridoces com frutas (manga, pera e uva passa), encontraram valores para intenção de compra, semelhantes a esse trabalho, sendo que estes variaram de 3,80 a 4,06, sendo classificadas como bem aceitas e apresentando potencial de mercado. Ramos et al. (2019) ao trabalharem com molho chutney de maçã com figo observaram intenção de compra semelhante a encontrada nesse estudo, com valores variando entre 3,00 e 4,00, sendo a amostra com maior quantidade de figo a que apresentou a maior intenção de compra.

Portanto, os molhos mostraram-se promissores para um lançamento no mercado, além de caracterizarem-se como produtos nos quais pode-se agregar uma infinidade de outros ingredientes visando melhorar a funcionalidade e a melhora da aceitação pelos consumidores.

\section{Considerações Finais}

Os molhos apresentaram cores claras e vivas, o que colaborou para a boa aceitação sensorial entre os provadores, no entanto fazem-se necessários ajustes na concentração da Goma Carragena para melhorar a aceitabilidade da consistência dos molhos. A Goma Xantana, nas condições empregadas no presente estudo, mostrou-se mais eficaz para o emprego em molho agridoce de abacaxi com pimenta, além da preferência pelos consumidores.

Sugere-se como continuidade a esse estudo avaliar diferentes proporções da goma xantana no molho agridoce de pimenta com abacaxi, bem como, o efeito da associação da goma xantana com outros espessantes com o intuito de avaliar o efeito sinérgico entre os mesmos.

\section{Agradecimentos}

Ao Instituto Federal de Educação, Ciência e Tecnologia do Sul de Minas Gerais, Campus Machado, pela disponibilização da infraestrutura.

\section{Referências}

Alves, L. A. B., \& Martins, C. B. (2017). Molho chutney: desenvolvimento e avaliação físico-química, microbiológica e sensorial. (Tese de Doutorado, Instituto Federal de Educação).

AUN, M. V., Mafra, C., Philippi, J. C., Kalil, J., Agondi, R. C., \& Motta, A. A. (2011). Aditivos em alimentos. Revista Brasileira de Alergia e imunopatologia, 34(5), 177-186.

Borges, R. B. G., Morais, R. A., Soares, C. M. S., Martins, G. A. S., Silva, J. F. M. (2020). Compostos bioativos do abacaxi (Ananás comosus) e da pimenta dedo de moça (Capsicum baccatum) e sua correlação com atividade antioxidante. Research, Society and Development, 9(7), e71973210-e71973210. Recuperado de doi:http://dx.doi.org/10.33448/rsd-v9i7.3210. 
Research, Society and Development, v. 10, n. 1, e32010111871, 2021

(CC BY 4.0) | ISSN 2525-3409 | DOI: http://dx.doi.org/10.33448/rsd-v10i1.11871

Brasil, F. I. (2010). Estabilizantes. Revista Food Ingredientes Brasil, (14), 42-48.

Carvalho, S. I. C., Ribeiro, C. S., Henz, G. P., \& Reifschneider, F. J. B. (2009). 'BRS Mari': Nova cultivar de pimenta dedo-de-moça para processamento. Horticultura Brasileira, Brasilia, 27(4), 571-573.

Dos Santos, A. T. V., Pereira, I. C., da Silva Oliveira, R. E., de Jesus, L. R. C., Pinheiro, I. B. L., \& de Medeiros Júnior, F. C. (2019). Elaboração de produto tipo brigadeiro a base da cenoura (Daucus carota L.). Research, Society and Development, 8(7), 14.

Ferreira, D. S., \& Sisvar, F. (2008). Um programa para análises e ensino de estatística. In Revista Científica Symposium, Lavras, (Vol. 6, No. 2, pp. 36-41).

Food, F. A. O. (2018). Agriculture Organization of the United Nations Statistical Databases. Recuperado de http//faostat. fao. org/site/339 default. aspx.

Funami, T., Kataoka, Y., Omoto, T., Goto, Y., Asai, I., \& Nishinari, K. (2005). Effects of non-ionic polysaccharides on the gelatinization and retrogradation behavior of wheat starch. Food Hydrocolloids, 19(1), 1-13. Recuperado de http://dx.doi.org/10.1016/j.foodhyd.2004.04.024

Instituto Americano De Culinária. Chef profissional. (2011) Tradução de Renata Lucia Bottini. 4. ed. São Paulo: Senac editoras.

Lainetti, A. M. D. S. (2017). Elaboração de Geleia de Abacaxi com Pimenta. (Dissertação de bacharelado, Universidade Tecnológica Federal do Paraná).

Minim, V. P. R., \& Reis, R. C. (2006). Testes de Aceitação. Minim, VP R. Análise Sensorial: Estudos com consumidores. Viçosa: UFV, 67-83.

Monteiro, C. L. B. (1984). Técnicas de avaliação sensorial. In Técnicas de avaliação sensorial (pp. 101-101).

Neres, J. P. G., de Souza, R. L. A., \& Bezerra, C. F. (2015). Iogurte com polpa e farinha da casca do abacaxi. Revista do Instituto de Laticínios Cândido Tostes, $70(5), 262-269$

Pereira, A. S., Shitsuka, D. M., Parreira, F. J., \& Shitsuka, R. (2018). Metodologia da pesquisa científica.[e-book]. Santa Maria: UAB/NTE/UFSM. Recuperado de https://repositorio.ufsm.br/bitstream/handle/1/15824/Lic_Computacao_Metodologia-Pesquisa-Cientifica.pdf?sequence=1

Quirino, C. D. S. (2017). Desenvolvimento e análises de qualidade do molho obtido a partir da polpa do mangostão amarelo (Garcinia xanthochymus). (Dissertação de bacharelado, Universidade Federal da Grande Dourados).

Rabelo, D. M., \& Nascimento, A. L. (2018). Desenvolvimento e Análise Sensorial da Geleia de Polpa e Casca de Abacaxi com Gengibre. Revista Acadêmica Conecta FASF, 3(1).

Ramos, A. C., Ferreira, A., Sousa, B. A. D. L., Pereira, E., Rodrigues, N., \& Pereira, J. A. (2019). Chutney: um aprovisionamento agridoce. In $2^{\circ}$ Congresso Luso-Brasileiro de Horticultura (No. 33, pp. 167-173). Universidade de São Paulo.

Sidra, I. (2014). Instituto Brasileiro de Geografia e Estatística. Sistema IBGE de Recuperação Automática. Recuperado em 04 junho, 2020 , de https://sidra.ibge.gov.br/tabela/5457\#resultado.

Valdez, B. (Ed.). (2012). Food Industrial Processes: Methods and Equipment. BoD-Books on Demand.

Véras, A. O. M. (2010). Secagem de pimenta dedo-de-moça (Capsicum baccatum var. pendulum) em secador convectivo horizontal. 